\title{
Design and Fabrication of a Novel Ultra Compact Microstrip Diplexer Using Interdigital and Spiral Cells
}

\author{
Salah I. Yahya ${ }^{1,2}$, Abbas Rezaei $^{3}$ \\ ${ }^{1}$ Department of Communication and Computer Engineering, Cihan University-Erbil, \\ Erbil, Iraq \\ ${ }^{2}$ Department of Software Engineering, Faculty of Engineering, Koya University, \\ Koya KOY45, Iraq \\ ${ }^{3}$ Department of Electrical Engineering, Kermanshah University of Technology, \\ Kermanshah, Iran
}

\begin{abstract}
A dual-band bandpass-bandpass microstrip diplexer with very small size and good performance is designed in this work. The proposed diplexer has a novel structure which is introduced for the $1^{\text {st }}$ time in this paper. In comparison with the previously reported diplexers, it occupies the most compact size of $0.002 \lambda \mathrm{g}^{2}\left(226.7 \mathrm{~mm}^{2}\right)$, fabricated on $0.787 \mathrm{~mm}$ dielectric substrate height. The resonance frequencies of the presented diplexer are located at $0.76 \mathrm{GHz}$ and $1.79 \mathrm{GHz}$ making it suitable for the global system for mobile communications applications. It has a wide flat channels with two fractional bandwidths (FBWs) of $41.1 \%$ and $50 \%$. Another feature of the proposed diplexer is its ability to suppress the harmonics. It can attenuate the $1^{\text {st }}-7^{\text {th }}$ harmonics. Moreover, it has low insertion losses and low group delays at both channels, whereas the isolation and return losses are acceptable. Finally, the proposed diplexer is fabricated and measured to verify the simulation results, where a good agreement between the simulation and measurement results is obtained.
\end{abstract}

Index Terms-Compact size, Diplexer, Low loss, Microstrip, Harmonics, Group delay.

\section{INTRODUCTION}

Dual-band microstrip diplexers are three-port passive components. They can be used for separating desired radiofrequency (RF) signals and delivering them through two channels (Yahya, Rezaei and Noori, 2020). The structure, performance, and designing method of several microstrip dual-band bandpass-bandpass diplexers have been reviewed in Yahya, Rezaei and Noori, 2020. Several types of microstrip structures have been utilized to design of dual-band bandpass-bandpass diplexers for modern

\section{ARO-The Scientific Journal of Koya University}

Vol. IX, No.1 (2021), Article ID: ARO.10819, 06 pages

DOI:10.14500/aro.10819

Received 20 April 2021; Accepted: 22 June 2021

Regular research paper: Published: 30 June 2021

Corresponding author's e-mail: salah.ismaeel@koyauniversity.org

Copyright (C) 2021 Salah I. Yahya and Abbas Rezaei. This is an openaccess article distributed under the Creative Commons Attribution License. communication systems (Guan, Yang, Liu and Zhu, 2014; Bukuru and Song, 2015; Chen, Zhu, Bu and Cheng, 2015; Peng and Chiang, 2015; Xiao, Zhu, Li, Tian and Ma, et al., 2015; Huang, Wang, Zhu and $\mathrm{Wu}, 2016$; Jun-Mei, Zhou and Cao, 2016; Salehi, Keyvan, Abiri and Noori, 2016; Bui, Vuong, Allard, Verdier and Benech, 2017; Feng, Zhang and Che, 2017; Noori and Rezaei, 2017a; Noori and Rezaei, 2017b; Rezaei, Noori and Mohamadi, 2017; Rezaei and Noori, 2018a; Rezaei and Noori, 2018b; Rezaei and Noori, 2018c; Danaeian, 2019; Kumar and Upadhyay, 2019; Rezaei, Yahya and Jamaluddin, 2019; Rezaei and Mohammadi, 2019; Rezaei, Noori and Mohammadi, 2019; Yahya, Rezaei and Noori, 2020; Yahya, Rezaei and Nouri, 2020; Rezaei, Yahya and Jamaluddin, 2020). However, occupying very large implementation areas are the common disadvantage of these diplexers. On the other hand, all of these diplexers have narrow channels, which make some of them suitable for only narrowband applications. Another common disadvantage of the above reported diplexers is their undesired harmonics. The coupling structures have been used in majority of these diplexers. Coupled meandrous cells in Rezaei, Yahya, Noori, and Jamaluddin, 2019a, coupled open-loop in Xiao, Zhu, Li, Tian and Ma, 2015, coupled step impedance and low impedance sections in Noori and Rezaei, 2017a, coupled spirals in Bukuru and Song, 2015, and coupled E-shape cells in Guan, Yang, Liu and Zhu, 2014 have been utilized. Similar to Rezaei, Yahya, Noori, and Jamaluddin, 2019a, the coupled meandrous cell has been used in Rezaei, Noori and Mohammedi, 2017. The proposed diplexers in Rezaei, Yahya, Noori, and Jamaluddin, 2019a; Xiao, Zhu, Li, Tian and Ma, 2015; Noori and Rezaei, 2017; Bukuru and Song, 2015; Guan, Yang, Liu and Zhu, 2014; Rezaei, Noori and Mohamadi, 2017, could not attenuate harmonics, where for the best case, they could only suppress the $3^{\text {rd }}$ harmonic. The other problems of the previous diplexers are poor selectivity in Rezaei, Yahya, Noori, and Jamaluddin, 2019a, high insertion losses in Xiao, Zhu, Li, Tian and Ma, 2015; Noori and Rezaei, 2017; Bukuru and Song, 2015; Guan, Yang, Liu and 
Zhu, 2014, undesired isolation between channels in Noori and Rezaei, 2017, and non-flat channels in Rezaei, Noori and Mohamadi, 2017. Despite this fact that the passband flatness and group delay are two important factors for filters and diplexers, majority of the previously reported diplexers did not pay attention to these problems. Meanwhile, there is some information about the flatness in Noori and Rezaei, 2017, which show that it has two non-flat channels with high group delays. In Noori and Rezaei, 2017b, an approximated equivalent $L C$ circuit of coupled lines has been analyzed to design of a microstrip diplexer for wireless applications. This diplexer has poor frequency selectivity at the upper channel. The interdigital cells in Bui, Vuong, Allard, Verdier and Benech, 2017, coupled triangular open-loop resonators in Salehi, Keyvan, Abiri and Noori, 2016, and engraved semi-patch cells in Rezaei and Noori, 2018a have been used to obtain three microstrip diplexers with low insertion losses for RF energy harvesting. Meanwhile, the introduced diplexer in Salehi, Keyvan, Abiri and Noori, 2016, could suppress harmonics from $1^{\text {st }}$ up to $4^{\text {th }}$ harmonics. In Chen, Zhu, $\mathrm{Bu}$ and Cheng, 2015, slot line-loaded microstrip ring resonator, in Rezaei and Noori, 2018b, the spiral and engraved cells, in Rezaei, Noori and Mohammadi, 2019, coupled meandrous structure, similar to Rezaei, Yahya and Jamaluddin, 2019; Rezaei, Noori and Mohammedi, 2017, and in Huang, Wang, Zhu and $\mathrm{Wu}, 2016$, coupled stubloaded U-shape cell have been used. The reported diplexer in Chen, Zhu, Bu and Cheng, 2015, has high insertion loss problem where the passbands in Rezaei and Noorin, $2018 \mathrm{~b}$, are very narrow. The achievements of the proposed diplexer in Rezaei, Yahya, Noori and Jamaluddin, 2019b, are low insertion losses at both channels and relatively wider fractional bandwidths (FBWs), whereas it is designed based on coupled step impedance cells. A diplexer using microstrip half- and quarter-wavelength resonators has been proposed in Jun-Mei, Zhou and Cao, 2016. In Peng and Chiang, 2015, new types of dual-mode ring filters have been used to design of two diplexers. These two diplexers have very high insertion losses at their channels. A novel diplexer based on a new form of coupled triangular microstrip structure has been proposed in Rezaei and Noori, 2018c, for L-band applications. This diplexer has low loss, narrow channels, and $3^{\text {rd }}$ attenuated harmonic. In Feng, Zhang and Che, 2017, the folded open-loop ring resonators have been utilized to obtain a microstrip diplexer with high isolation. In Yahya, Rezaei and Nouri, 2020, an artificial neural network (ANN) has been used to design of a compact microstrip diplexer. In Roshani and Roshani, 2019, a compact and sharp diplexer with bended lines has been introduced for the global system for mobile communications and long-term evolution (LTE) applications. In Kumar and Upadhyay, 2019, a via-free composite right/left-handed transmission line-based diplexer has been introduced for WiMAX and WLAN applications. In $\mathrm{Xu}, \mathrm{Yu}$ and Chen, 2021, a tunable microstrip diplexer with multiple transmission zeroes has been presented using common element-loaded uniform impedance resonator (UIR). In Chen, et al., 2021, a novel circuit model has been presented to design of microstrip multifunction integrated diplexers with frequency selection, frequency division, and power division functions.

This work presents the design of a microstrip diplexer, which has the most compact size, the widest FBWs, low insertion losses, and the best stopband rejection, whereas the other diplexer parameters are acceptable. The proposed diplexer has a novel microstrip structure, which is presented for the $1^{\text {st }}$ time in this work. It is composed of the interdigital and spiral cells.

\section{Design AND ANALYsis}

To create a passband channel, we used the spiral and interdigital cells with the inductance and capacitance features, respectively. The advantage of using the spiral cells is saving the size significantly. Meanwhile, by optimizing the structure, the interdigital cell can be used as a compact capacitor. Accordingly, we proposed a microstrip resonator as depicted in Fig. 1a. It includes two spiral cells with different dimensions, which are integrated by an interdigital structure. Thus, this structure can create a passband channel. Fig. 1b shows an approximated equivalent $L C$ circuit of the proposed resonator, where $L_{1}$ and $L_{2}$ are related to the spiral cells 1 and 2, respectively. As mentioned above, the middle interdigital cell is a capacitor which is shown by $C$. By removing the insignificant inductors and capacitors related to the bent and steps in widths, our $L C$ circuit can be simplified.

The ABCD matrix of the proposed resonator is calculated as follows:

$$
T=\left[\begin{array}{ll}
A & B \\
C & D
\end{array}\right]=\left[\begin{array}{cc}
1 & j \omega\left(L_{1}+L_{2}\right)+\frac{1}{j \omega C} \\
0 & 1
\end{array}\right]
$$

where, $\omega$ is an angular frequency. As presented in the following equation, the reflection coefficient can be obtained:

$$
\begin{aligned}
& \Gamma=\frac{A+B-C-D}{A+B+C+D}=\frac{j \omega\left(L_{1}+L_{2}\right)+\frac{1}{j \omega C}}{2+j \omega\left(L_{1}+L_{2}\right)+\frac{1}{j \omega C}} \\
& =\frac{1-\omega^{2} C\left(L_{1}+L_{2}\right)}{2 j \omega C-\omega^{2} C\left(L_{1}+L_{2}\right)+1}
\end{aligned}
$$

For $\Gamma=0$, we can obtain a perfect matching.

$$
\Gamma=0 \Rightarrow \omega_{m}=\frac{1}{\sqrt{C\left(L_{1}+L_{2}\right)}}
$$

Where, $\omega_{m}$ is the angular frequency with perfect matching. On the other hand, we can calculate the input impedance $\left(Z_{\text {in }}\right)$ from its equivalent $L C$ circuit of the proposed resonator:

$$
\operatorname{Zin}=\left(L_{1}+L_{2}\right) j \omega+\frac{1}{j \omega C}
$$

The resonance frequency can be obtained from (4) by setting $Z_{\text {in }}=0$. Therefore, the angular resonance frequency $\left(\omega_{r}\right)$ is:

$$
\omega_{r}=\frac{1}{\sqrt{C\left(L_{1}+L_{2}\right)}}
$$


It is clear that $\omega_{r=\omega_{m}}$ which proves we have a perfect matching at the resonance frequency. Consequently, our resonator has a low loss inherently. To miniaturize the resonator size, we can optimize its dimensions by changing the widths of microstrip lines. Since we have only a resonance frequency, our resonator is a single mode without harmonics. We can see harmonics in the frequency response of the resonators with several resonance frequencies. Some of the calculated resonance frequencies may represent

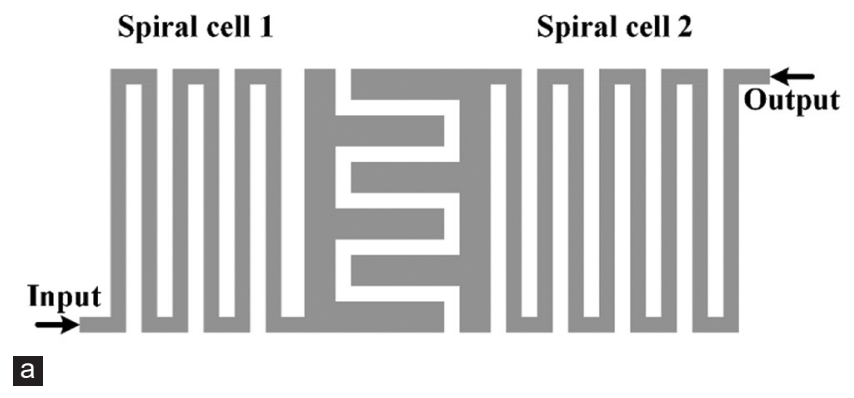

b

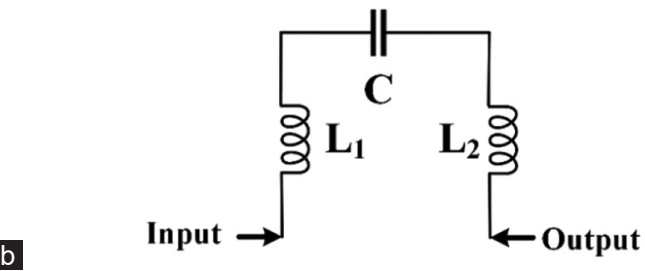

Fig. 1. The presented resonator (a) layout, (b) an approximated $L C$ circuit. unwanted harmonics (Salehi and Noori, 2015), therefore, our resonator is able to suppress the harmonics inherently. By knowing this information about resonator behavior, we can optimize the dimensions and find the physical dimensions of our proposed structures. Based on the proposed resonator, two bandpass filters (BPFs) are designed and presented in Fig. 2a and b, which we named them BPF1 and BPF2, respectively. The dimensions of both BPFs are in $\mathrm{mm}$. As depicted in Fig. 2a and b, the spiral and interdigital structures are used similar to the designed resonator. The proposed filters are simulated by ADS software on a RT/ duroid 5880 substrate with $\varepsilon_{\mathrm{r}}=2.2, h=31 \mathrm{mil}$, and 0.0009 loss tangent. The frequency responses of BPF1 and BPF2 are shown in Fig. 2c and d, respectively. As shown in these figures, BPF1 creates a channel from $590 \mathrm{MHz}$ to $900 \mathrm{MHz}$ with an operational frequency at $f_{\mathrm{ol}}=740 \mathrm{MHz}$. It attenuates the harmonics up to $\left.3.37 f_{\mathrm{ol}} 2.5 \mathrm{GHz}\right)$, whereas the maximum harmonic level is $20 \mathrm{~dB}$. Moreover, it has $0.1 \mathrm{~dB}$ low insertion loss. The frequency response of BPF2 shows that it operates at $f_{\mathrm{o} 2}=1.85 \mathrm{GHz}$ with $0.049 \mathrm{~dB}$ insertion loss and $22.5 \mathrm{~dB}$ return loss. This very low insertion loss verifies the perfect matching at the resonance frequency. The $-3 \mathrm{~dB}$ cutoff frequencies of BPF2 are $1.41 \mathrm{GHz}$ and 2.3 GHz so that it has a wide flat channel. Using BPF1 and BPF2, we designed a compact microstrip diplexer. The layout configuration of the proposed diplexer is shown in Fig. 3. The proposed diplexer is formed by integrating BPF1 and BPF2 so that the other dimensions are exactly similar to Fig. $2 \mathrm{a}$ and $\mathrm{b}$.
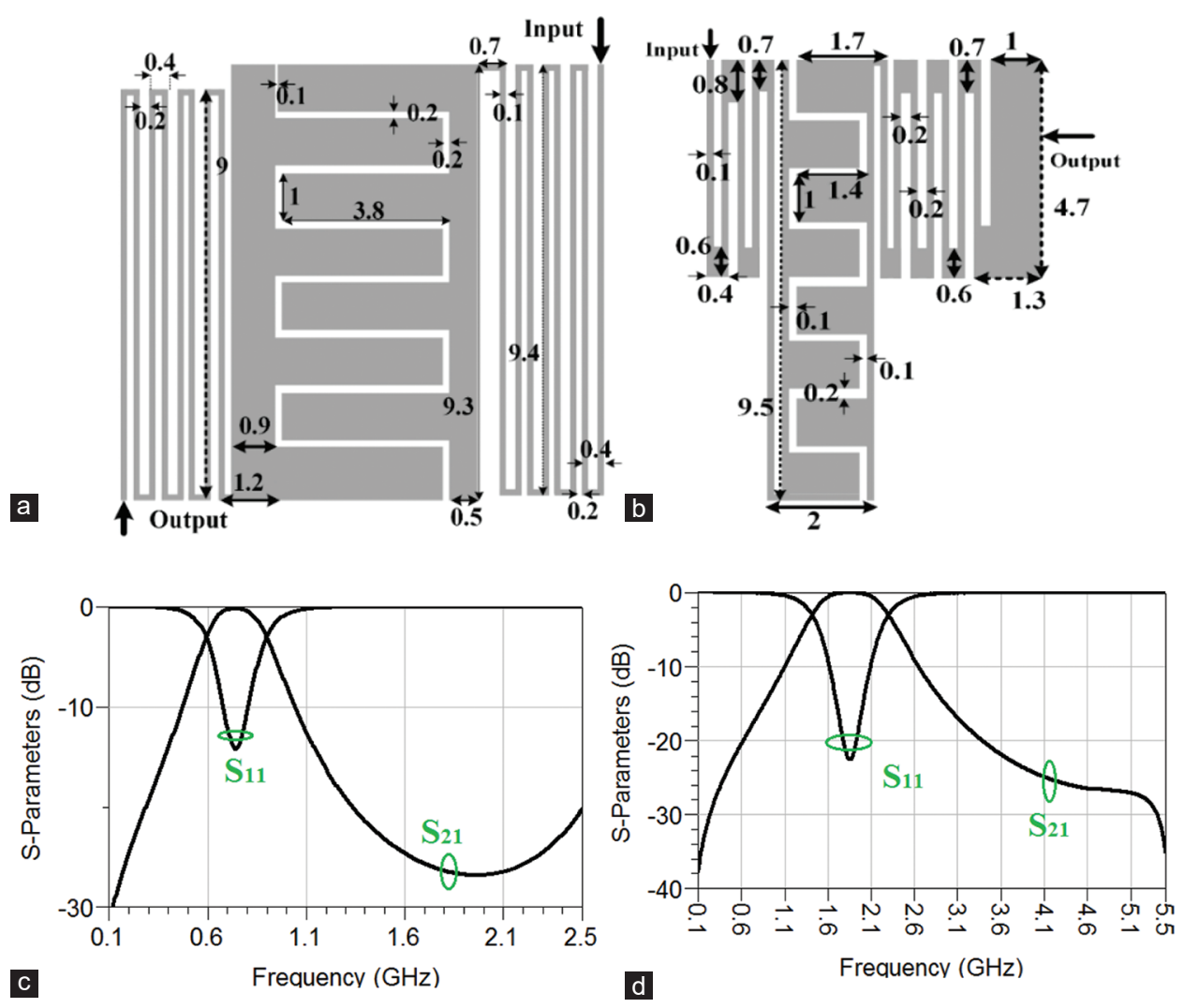

Fig. 2. (a) BPF1, (b) BPF2, (c) frequency response of BPF1, (d) frequency response of BPF2. 


\section{RESUlts, Discussion, AND COMPARISON}

The designed diplexer is simulated by the full-wave EM simulator of Advanced Design System (ADS) software. It is designed, simulated, and fabricated on a RT/Duroid 5880 substrate with $\varepsilon_{\mathrm{r}}=2.2, h=31 \mathrm{mil}$, and 0.0009 loss tangent. The fabricated structure is measured by Agilent network analyzer N5230A. The simulated and measured $S_{21}$ and $S_{31}$ of the proposed diplexer are depicted in Fig. 4a, whereas Fig. 4b shows the simulated and measured common port return loss and isolation between the channels. The first channel is from $615 \mathrm{MHz}$ to $925 \mathrm{MHz}$ with $41.1 \% \mathrm{FBW} \%$. The resonance frequency of this channel is located at $f_{\mathrm{rl}}=755 \mathrm{MHz}$, where it has $0.169 \mathrm{~dB}$ and $15.7 \mathrm{~dB}$ simulated insertion and return losses, respectively. The resonance frequency of the upper channel is $f_{\mathrm{r} 2}=1.79 \mathrm{GHz}$, whereas it has two $-3 \mathrm{~dB}$ cutoff frequencies at $1.4 \mathrm{GHz}$ and $2.3 \mathrm{GHz}$. Similar to the lower channel, the upper channel is wide and flat with 50\% FBW\%.

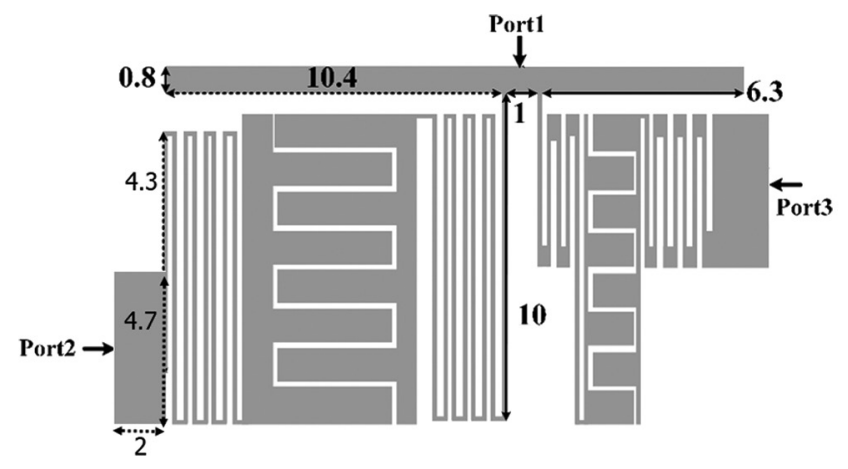

Fig. 3. The proposed diplexer layout.

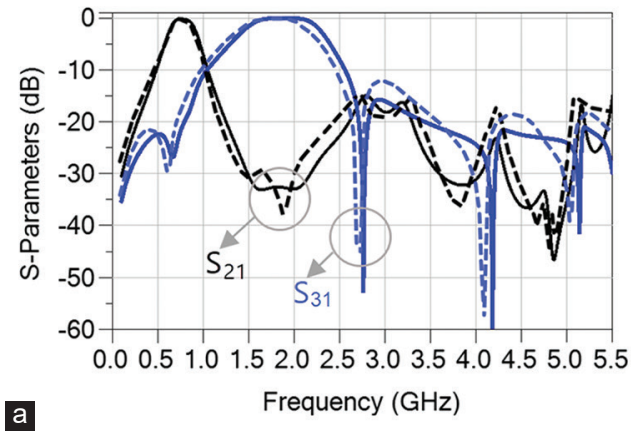

The simulated insertion and return losses of this channel are better than $0.08 \mathrm{~dB}$ and $24.8 \mathrm{~dB}$, respectively. Due to the copper and junction losses in the fabricated diplexer, the measured losses are a little higher than the simulation results. As presented in Fig. 4a, the harmonics are attenuated up to $5.5 \mathrm{GHz}\left(7.28 f_{\mathrm{r} 1}\right)$. It means that the proposed diplexer can suppress $1^{\text {st }}$ up to $7^{\text {th }}$ harmonics with the maximum harmonic level $14.92 \mathrm{~dB}$. The isolation between the channels is better than $19.98 \mathrm{~dB}$ from $\mathrm{DC}$ to $5.5 \mathrm{GHz}$, which is an acceptable value. The overall size of our compact diplexer is only $10.9 \mathrm{~mm} \times 20.8 \mathrm{~mm}\left(0.002 \lambda_{\mathrm{g}}{ }^{2}\right)$, where $\lambda_{\mathrm{g}}$ is the guided wavelength calculated at the first resonance frequency. Due to having SubMiniature Version A connectors and junction losses, the measured losses are a little higher than the simulated losses. A photograph of the fabricated diplexer is presented in Fig. 4b. To show the features of this work, we compared it with the previously reported diplexers. The comparison results are presented in Table I, where $f_{\mathrm{r}}$, IL, and FBW are the resonance frequency, insertion loss, and FBWs, respectively. Furthermore, the indexes 1 and 2 are related to the $1^{\text {st }}$ and $2^{\text {nd }}$ channels, respectively. As shown in the comparison table, our diplexer is the most compact diplexer and it is very smaller than the other previously reported diplexers. Moreover, it has the widest FBWs, low simulated insertion losses at both channels, and the best harmonic attenuation.

Fig. 5a and $\mathrm{b}$ shows the group delays of the lower and upper channels, respectively. As depicted in Fig. 5, the group delays at the first and second channels are lower than $1.73 \mathrm{~ns}$ and $0.79 \mathrm{~ns}$, respectively. Despite the importance of

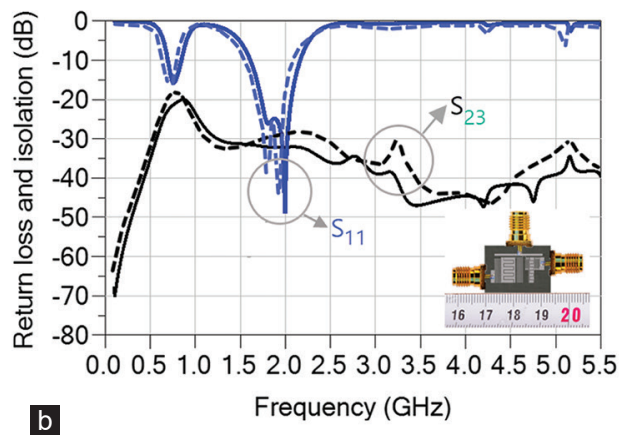

Fig. 4. (a) Simulated (solid line) and measured (dotted line) $S_{21}$ and $S_{31}$, (b) simulated and measured common port return loss and isolation between the channels and the fabricated diplexer.

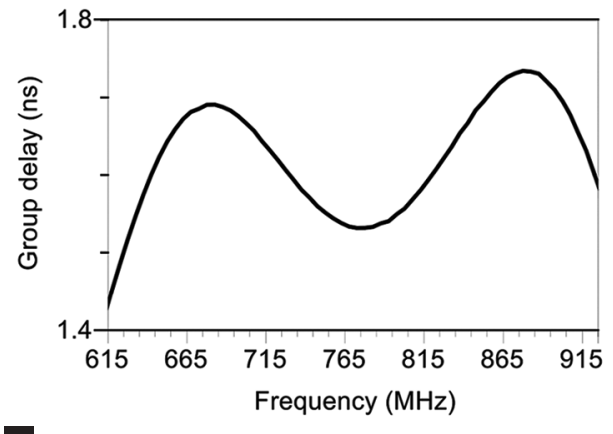

a

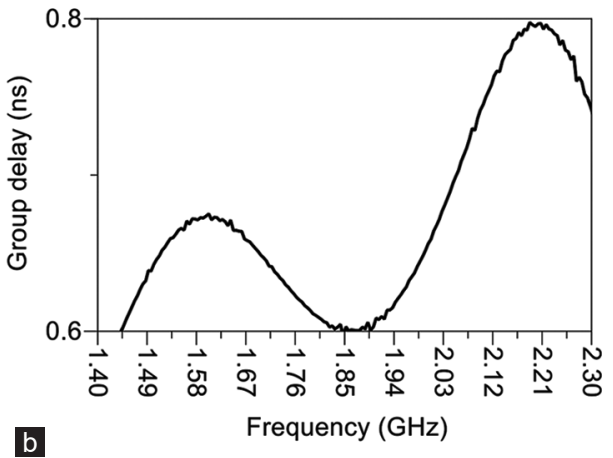

b

Fig. 5. Group delays at the (a) first channel, (b) second channel. 
TABLE I

COMPARISON BETWEEN THIS DIPLEXER AND THE PREVIOUSLY REPORTED DIPLEXERS

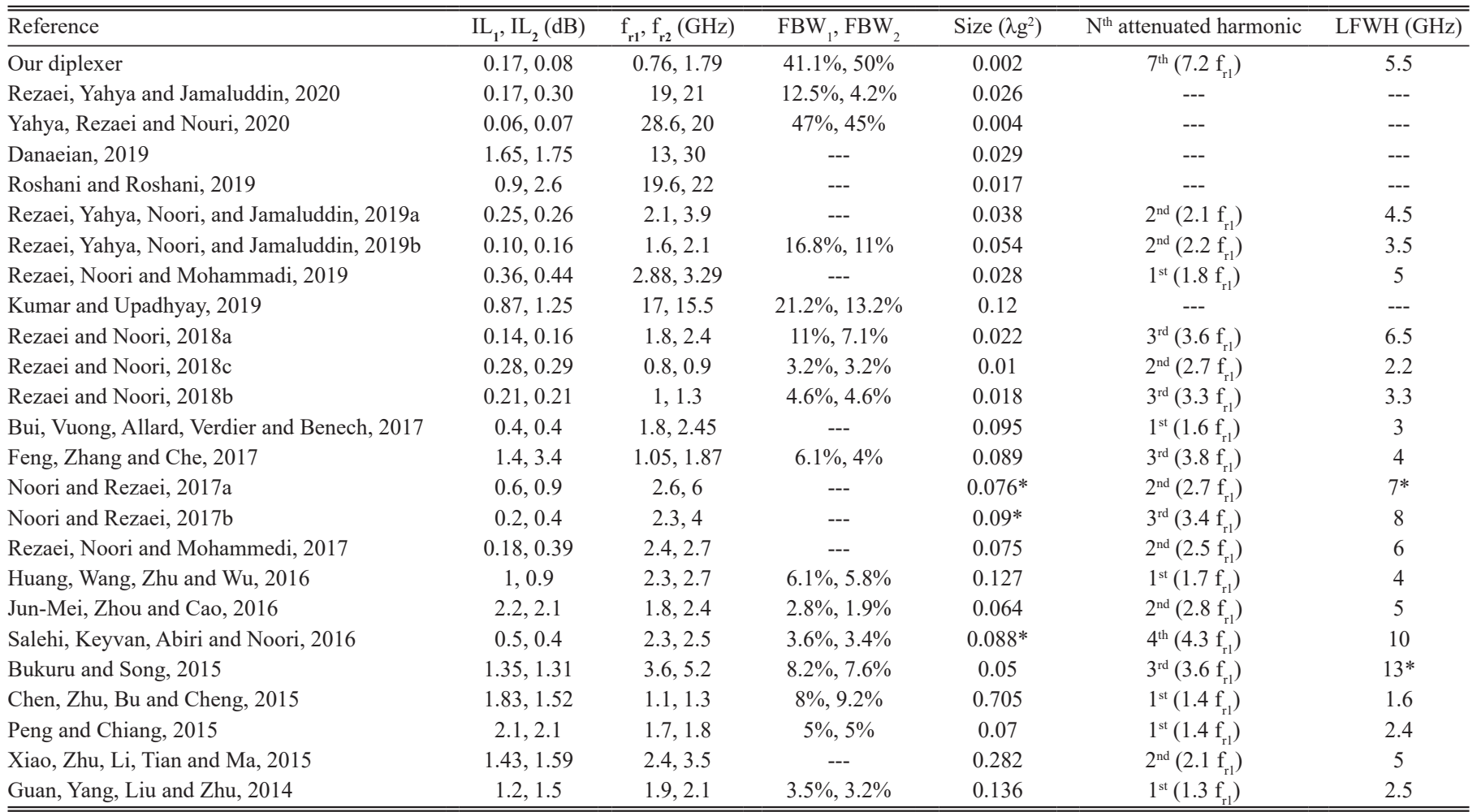

LFWH: Last frequency without harmonics; *: Approximated values

TABLE II

THE MAXIMUM GROUP DELAYS COMPARISON

\begin{tabular}{lcc}
\hline \hline References & $\begin{array}{c}\text { Maximum group delay } \\
\text { at first channel }\end{array}$ & $\begin{array}{c}\text { Maximum group delay at } \\
\text { the second channel }\end{array}$ \\
\hline This work & $1.73 \mathrm{~ns}$ & $0.79 \mathrm{~ns}$ \\
Rezei, et al., 2019a & $4 \mathrm{~ns}$ & $1.8 \mathrm{~ns}$ \\
Noori and Rezaei, 2017a & $3.15 \mathrm{~ns}$ & $2.98 \mathrm{~ns}$ \\
Rezaei and Noori, 2018a & $3 \mathrm{~ns}$ & $3.14 \mathrm{~ns}$ \\
Rezaei, Yahya, Noori, and & $2.2 \mathrm{~ns}$ & $2.6 \mathrm{~ns}$ \\
Jamaluddin, 2019b & & $4.25 \mathrm{~ns}$ \\
Rezaei and Noori, 2018b & $4.5 \mathrm{~ns}$ & \\
\hline \hline
\end{tabular}

*: Approximated value

group delay, it is not included in many reported diplexers. However, some of the previous works mentioned the group delay that we compared them with our work in Table II. As depicted in Table II, the lowest group delays at both channels are obtained in this work.

\section{CONCLUSION}

This paper presents a novel microstrip resonator with several features for the $1^{\text {st }}$ time in this work. The proposed microstrip resonator includes a step impedance interdigital cell connected to the spiral structures. We used the presented resonator to design of two compact BPFs. By integrating the proposed BPFs using a simple matching circuit, a highperformance microstrip diplexer was presented. This diplexer has the most compact size in comparison with the previous works. It has two flat channels with the lowest group delays better than 1.73 ns. Furthermore, our diplexer has the widest FBW and the best harmonic suppression.

\section{REFERENCES}

Bui, D.H.N., Vuong T.P., Allard B., Verdier J. and Benech P., 2017. Compact low-loss microstrip diplexer for RF energy harvesting. Electronics Letters, 53(8), pp.552-554.

Bukuru, D. and Song, K., 2015. Compact wide-stopband planar diplexer based on rectangular dual spiral resonator. Microwave and Optical Technology Letters, 57(1), pp.174-178.

Chen, C.F., Zhou, K.W., Chen, R.Y., Tseng, H.Y., He, Y.H., Li, W.J. and Weng, J.H., 2021. Design of Microstrip multifunction integrated diplexers with frequency division, frequency selection, and power division functions. IEEE Access, 9, pp.53232-53242.

Chen, D., Zhu, L., Bu, H. and Cheng, C.H., 2015. A novel planar diplexer using slot line-loaded microstrip ring resonator. IEEE Microwave and Wireless Components Letters, 25(11), pp.706-708.

Danaeian, M., 2019. Miniaturized half-mode substrate integrated waveguide diplexer based on SIR-CSRR unit-cell. Analog Integrated Circuits and Signal Processing, 102, pp.555-561.

Feng, W., Zhang, Y. and Che, W., 2017. Tunable dual-band filter and diplexer based on folded open loop ring resonators. IEEE Transactions on Circuits and Systems, 64(9), pp.1047-1051.

Guan, X., Yang, F., Liu, H. and Zhu, L., 2014. Compact and high-isolation diplexer using dual-mode stub-loaded resonators. IEEE Microwave and Wireless Components Letters, 24(6), pp.385-387.

Huang, F., Wang, J., Zhu, L. and Wu, W., 2016. Compact microstrip balun diplexer using stub-loaded dual-mode resonators. IET Electronic Letters, 52, pp.1994-1996. 
Jun-Mei, Y., Zhou, H.Y. and Cao, L.Z., 2016. Compact diplexer using microstrip half- and quarter wavelength resonators. Electronics Letters, 52(19), pp.16131615.

Kumar, A. and Upadhyay, D., 2019. A compact planar diplexer based on viafree CRLH TL for WiMAX and WLAN applications. International Journal of Microwave and Wireless Technologies, 11, pp.130-138.

Noori, L. and Rezaei A., 2017a. Design of a microstrip dual-frequency diplexer using microstrip cells analysis and coupled lines components. International Journal of Microwave and Wireless Technologies, 9(7), pp.1467-1471.

Noori, L. and Rezaei, A., 2017b. Design of a microstrip diplexer with a novel structure for WiMAX and wireless applications. AEU-International Journal of Electronics and Communications, 77, pp.18-22.

Peng, H. and Chiang, Y., 2014. Microstrip diplexer constructed with new types of dual-mode ring filters. IEEE Microwave and Wireless Components Letters, 25(1), pp.7-9.

Rezaei, A. and Noori, L., 2018a. Compact low-loss microstrip diplexer using novel engraved semi-patch cells for GSM and WLAN applications. AEUInternational Journal of Electronics and Communications, 87, pp.158-163.

Rezaei, A. and Noori, L., 2018b. Miniaturized microstrip diplexer with high performance using a novel structure for wireless L-band applications. Wireless Networks, 26, pp.1795-1802.

Rezaei, A. and Noori, L., 2018c. Novel compact microstrip diplexer for GSM applications. International Journal of Microwave and Wireless Technologies, 10(3), pp.313-317.

Rezaei, A., Noori, L. and Mohamadi, H., 2017. Design of a novel compact microstrip diplexer with low insertion loss. Microwave and Optical Technology Letters, 59(7), pp.1672-1676.

Rezaei, A., Noori, L. and Mohammadi, H., 2019. Design of a miniaturized microstrip diplexer using coupled lines and spiral structures for wireless and WiMAX applications. Analog Integrated Circuits and Signal Processing, 98, pp.409-415.
Rezaei, A., Yahya S.I., Noori, L. and Jamaluddin, M.H., 2019a. Design and fabrication of a novel compact low-loss microstrip diplexer for WCDMA and WiMAX applications. Journal of Microwaves, Optoelectronics and Electromagnetic Applications, 18(4), pp.482-491.

Rezaei, A., Yahya, S.I. and Jamaluddin M.H., 2020. A novel microstrip diplexer with compact size and high isolation for GSM applications. AEU-International Journal of Electronics and Communications, 114, p.153018.

Rezaei, A., Yahya, S.I., Noori L. and Jamaluddin, M.H., 2019b. Design of a novel wideband microstrip diplexer using artificial neural network. Analog Integrated Circuits and Signal Processing, 101(1), pp.57-66.

Roshani, S. and Roshani S., 2019. Design of a very compact and sharp bandpass diplexer with bended lines for GSM and LTE applications. AEU-International Journal of Electronics and Communications, 99, pp.354-360.

Salehi, M and Noori, L., 2015. Miniaturized microstrip bandpass filters using novel stub loaded resonator. ACES Journal, 30(6), pp.692-697.

Salehi, M.R., Keyvan, S., Abiri E. and Noori L., 2016. Compact microstrip diplexer using new design of triangular open loop resonator for $4 \mathrm{G}$ wireless communication systems, AEU-International Journal of Electronics and Communications, 70(7), pp.961-969.

Xiao, J. K., Zhu M., Li Y., Tian L. and Ma J.G., 2015. High selective microstrip bandpass filter and diplexer with mixed electromagnetic coupling, IEEE Microwave and Wireless Components Letters, 25(12), pp.781-783.

Xu L., Yu W. and Chen J.X., 2021. Unbalanced-/balanced-to-unbalanced diplexer based on dual-mode dielectric resonator. Access IEEE, 9, pp.53326-53332.

Yahya, S.I., Rezaei, A. and Noori, L., 2020. Design and performance of microstrip diplexers: A review. ARO-The Scientific Journal Koya University, 8(1), pp.38-49.

Yahya, S.I., Rezaei, A. and Nouri, L., 2020. The use of artificial neural network to design and fabricate one of the most compact microstrip diplexers for broadband L-band and S-band wireless applications. Wireless Networks, 27, pp.663-676. 